\title{
SAGES clinical spotlight review: endoluminal treatments for gastroesophageal reflux disease (GERD)
}

\author{
C. Daniel Smith
}

Received: 7 May 2013/Accepted: 7 May 2013/Published online: 21 May 2013

(C) Springer Science+Business Media New York 2013

Auyang, Carter, Rauth, and Fanelli, along with the members of the SAGES Guidelines Committee, have produced a comprehensive, clear review of available medical literature on the topic of endoluminal treatments for gastroesophageal reflux disease (GERD). This represents an objective, fair, and credible assessment of a diverse body of literature covering a complex and thorny topic. The conclusions and recommendations are invaluable to the physician and surgeon caring for patients with GERD, helping guide not only their advice to patients, but also their own understanding of these offerings and where they fit in today's treatment of the patient with GERD.

I'd like to expand on two carefully chosen words out of the preceding paragraph, complex and thorny, and challenge us to consider better ways to assess new technology so as to achieve an earlier understanding of whether novel technology has a role in our care of patients. First, this clinical spotlight review (CSR) tackles a complex clinical condition and its treatment, GERD. Although GERD as a disease is complex, its basic definition is not: it is simply abnormal reflux of gastric content into the esophagus due to an ineffective lower esophageal sphincter (LES) - a fairly clear definition of a mechanical defect. Yet it has taken decades for us to develop and understand a series of diagnostics to accurately diagnose GERD. Even after decades of study, there remain challenging diagnostic situations, such as how to diagnose clinically significant laryngopharyngeal reflux or how to identify and quantitate clinically significant nonacid reflux. With this, innovation around diagnostics for GERD continues. Many recent

C. D. Smith $(\bowtie)$

Department of Surgery, Mayo Clinic Florida, 4500 San Pablo Road, Jacksonville, FL 32224, USA

e-mail: smith.c.daniel@mayo.edu introductions provide new insights and ultimately will result in better care for GERD patients (e.g., impedance $\mathrm{pH}$ and high-resolution manometry). In contrast, our therapeutic options have changed little in the past decades: acid suppression with proton pump inhibitors and $\mathrm{H} 2$ blockers, or a half-century-old operation to augment the ineffective LES, the esophagogastric fundoplication (Nissen or Toupet fundoplication). Although the basis for acid suppression with medication has been well understood and accepted, the mechanism of action of the fundoplication - and more importantly translating a mechanism of action into a standardized technique to achieve consistent and good results-has remained elusive. Hence, achieving a true augmentation of the ineffective LES remains complex. With this as a backdrop, the development and advancement of endoluminal therapies has introduced a whole new level of complexity and confusion to GERD management, largely based on new hypothetical mechanisms of action added to an already poorly understood mechanism for how fundoplication works. For example, when considering these endoluminal therapies, how exactly does delivering radiofrequency energy to the LES alter LES function to control GERD, or can any totally endoluminal technique dependent on tissue fixation really create a durable plication or effect a true fundoplication?

Ideally, when assessing new technology, data associated with the development of these new devices will help answer some of these questions. This is where this topic gets thorny. As is true with many new surgical techniques introduced during the past 10-20 years, endoluminal treatments for GERD are oriented around new devices, and with this, these interventions are largely industry driven in their development and introduction into clinical practice. Industry's interest and development of new devices are essential to our ability to develop and implement new 
treatment for our patients, but industry's ability to pursue new devices is limited by the economics of getting a new device launched.

When industry brings a device to market, it can be a multimillion-dollar gamble with multiple hurdles between great innovation and a successful device. Not the least of these hurdles is the path to U.S. Food and Drug Administration (FDA) clearance or approval, but increasingly, FDA approval for a device is only the price of admission to a much murkier and high-risk path to technology adoption and ultimate reimbursement. There is not an infinite amount of money with which to effect exhaustive, prospective randomized control trials with longitudinal studies and long-term outcomes. This means moving a product into the market as efficiently as possible, sometimes with strong source data sufficient for the U.S. FDA, but far from published in peer-reviewed literature. This often leaves us with a device available for use but with limited devicespecific data. Perhaps not surprisingly, many of these new devices are considered experimental by payers, leaving the practitioner with virtually no possibility of actually using these new devices in the care of their patients. The challenge this presents is evident when we see what has happened to most innovation around GERD over the past 2 decades. This CSR covers two endoluminal treatments and devices, but there have been at least 4 others that have been launched in the past 10-15 years. The companies that launched these other devices are no longer in business, most often because as startups, they did not have the wherewithal to survive the long and uncertain process of gaining traction in a market where reimbursement is delayed until substantial long-term longitudinal data were available. Without such data, most payers refuse reimbursement for new technologies.

One might appropriately argue that this is precisely why we should wait for substantial and irrefutable data before reimbursing new procedures. Although I would agree in principle, in practice, we have a dysfunctional process that unnecessarily prolongs and bureaucratizes the steps between introduction of promising innovative technology and availability of this technology to patients. Often procedures and devices remain labeled as "experimental", claiming insufficient data from which to make a reimbursement determination; however, this label is often placed by administrative doctors who are outside of the specialty involving the technology and/or are no longer in the active practice of medicine. It is this barrier that is the immediate threat to our ability to bring innovative technology into practice, but the threat extends beyond the medical director of insurance companies.

We are at a troubling moment in surgery. Health care reform, cost containment, a shadow cast over industry's role in partnering with physicians and professional medical associations (PMAs) to develop new therapies, and an antiinnovation culture among many in Washington threaten the very innovations that have brought us the valuable changes in surgery over the past several decades (e.g., minimally invasive surgery). As the thought leaders and content experts in general and gastrointestinal surgery, it is incumbent on us, as well as PMAs such as SAGES, to provide guidance and leadership through these complex and thorny issues.

SAGES has already taken a stand in response to the growing impression that PMAs and industry relationships are conflicted and potentially corrupt $[1,2]$, and now, with this CSR providing a credible analysis of newer technology, SAGES has introduced a process where new devices and technologies can undergo a careful assessment combining published literature with expert opinion to facilitate a determination about where and how to use these technologies to help manage patients with GERD. Although the CSR process allows for an assessment in advance of a formal clinical practice guideline [3], it relies primarily on published data. Many new devices gain U.S. FDA approval through a premarket approval process whereby significant clinical data may be generated without peer-reviewed publication-data that are exhaustively reviewed by the FDA before a specialty panel before a device achieves FDA approval. These data are largely ignored until they are available in the peer-reviewed literature, despite the data conforming to the high scientific standards demanded by the FDA.

To further facilitate an early assessment of new devices and technology, SAGES has launched a technology and value assessment process where emerging technology that has achieved FDA clearance or approval can undergo a thorough assessment by a panel of experts to generate a technology and value assessment document. This document is intended to review and summarize all available data, including source data not yet in the peer-reviewed literature, and to provide an expert opinion on the safety, effectiveness, and in some cases value of a given technology. It is hoped that such a document will provide needed guidance for patients, physicians, and payers with regards to a new technology and will provide an early assessment that will permit decisions to be made about adoption and reimbursement early enough that patients can benefit and industry can continue to innovate.

Disclosures C. Daniel Smith is a paid consultant to Torax Medical, a company with a recently FDA approved device for the treatment of GERD.

\section{References}

1. Smith CD, Buyske J, Talamini MA (2009) Industry support and professional medical associations. JAMA 302(7):738-739 
2. Smith CD, MacFadyen B (2010) Industry relationships between physicians and professional medical associations: corrupt or essential? Surg Endosc 24:251-253
3. Institute of Medicine (2011) Clinical practice guidelines we can trust. Consensus report. http://www.iom.edu/Reports/2011/ClinicalPractice-Guidelines-We-Can-Trust.aspx. Accessed 1 Apr 2013 